\title{
The Rapid Assessment and Early Warning Models for COVID-19
}

\author{
Zhihua Bai $^{1,2} \cdot$ Yue Gong $^{3} \cdot$ Xiaodong Tian $^{1} \cdot$ Ying Cao $^{1} \cdot$ Wenjun $\mathrm{Liu}^{1,2,4} \cdot \mathrm{Jing} \mathrm{Li}^{1}$ (]
}

Received: 8 March 2020 / Accepted: 27 March 2020 / Published online: 1 April 2020

(C) Wuhan Institute of Virology, CAS 2020

\begin{abstract}
Human beings have experienced a serious public health event as the new pneumonia (COVID-19), caused by the severe acute respiratory syndrome coronavirus has killed more than 3000 people in China, most of them elderly or people with underlying chronic diseases or immunosuppressed states. Rapid assessment and early warning are essential for outbreak analysis in response to serious public health events. This paper reviews the current model analysis methods and conclusions from both micro and macro perspectives. The establishment of a comprehensive assessment model, and the use of model analysis prediction, is very efficient for the early warning of infectious diseases. This would significantly improve global surveillance capacity, particularly in developing regions, and improve basic training in infectious diseases and molecular epidemiology.
\end{abstract}

Keywords COVID-19 $\cdot$ SARS-CoV-2 $\cdot$ Models $\cdot$ Rapid assessment $\cdot$ Early warning

\section{Introduction}

Since the early twenty-first century, human beings have experienced several serious public health events caused by pathogens shared with wild or domestic animals, such as severe acute respiratory syndrome coronavirus (SARS$\mathrm{CoV}$ ), Middle East Respiratory Syndrome coronavirus (MERS-CoV), and influenza A (H1N1) virus et al. Emerging zoonoses pose a growing threat to global health, having caused hundreds of billions of dollars in economic losses in the past two decades (Karesh et al. 2012). The severe acute respiratory syndrome coronavirus 2 (SARSCoV-2) which can be deadly was first identified in Wuhan,

Zhihua Bai and Yue Gong have been contributed equally to this work.

Jing Li

1j418@163.com

1 CAS Key Laboratory of Pathogenic Microbiology and Immunology, Institute of Microbiology, Chinese Academy of Sciences, Beijing 100101, China

2 University of Chinese Academy of Sciences, Beijing 100049, China

3 National Science Library, Chinese Academy of Sciences, Beijing 100190, China

4 Institute of Microbiology, Center for Biosafety MegaScience, Chinese Academy of Sciences, Beijing 100101, China
China in December 2019 (Cheng and Shan 2020; Wu P et al. 2020b).

It evolved from wildlife and can cause fever and severe respiratory syndrome in human being which were named COVID-19 by the World Health Organization (WHO) (Huang et al. 2020; Xu et al. 2020). As of March 27, 2020, the still ongoing outbreak of around 82,098 confirmed cases and 3298 deaths in mainland China (http://2019ncov. chinacdc.cn/2019-nCoV/), and Most new coronavirus cases are now outside of China, with 200 countries reporting cases up to now. On January 30, 2020, the WHO announced that the epidemic constituted a public health emergency of international concern. These vulnerabilities emphasize the need for a systematic, preemptive approach that aims to prevent the spread, or even the initial emergence of pandemics. The systematic preemptive approach refers to the rapid assessment and an early warning of outbreaks of infectious diseases.

Early warning is an important measure to control and prevent outbreaks and epidemics of infectious diseases. The core is to find the abnormal distribution of infectious diseases and then estimate the risk of an outbreak. Its main work is to collect and analyze relevant information and data on the incidence of infectious diseases, and to explore the spatiotemporal transmission and epidemic laws of infectious diseases by using genomics, statistics and mathematical methods. It is indispensable to use mathematical methods to establish appropriate warning models in 
the early epidemic stage so that relevant departments can take timely response measures to prevent the development into a malignant state and reduce the degree of harm as far as possible. As recognized by the WHO, mathematical models, especially those that are timely, play a key role in informing evidence-based decisions by policy-makers (Morgan 2019).

\section{Prediction Models in Virus Traceability}

Whenever a new pathogen appears, its origin always causes widespread concern. Most of the emerging infectious diseases that affect humans are zoonotic, which can be traced back to some specific wildlife, but there have been few analytical tools to determine which host species may carry the next virus that will infect humans, or which viruses may cross species boundaries directly. However, with the advent of the era of big data, it no longer seems to be a difficult problem (Fig. 1).

\section{Prediction of the Potential of the Virus to Infect People}

In order to understanding patterns of viral diversity in wildlife and determinants of successful cross-species transmission, or spillover, Kevin et al. created a database of 2805 mammal-virus associations, including 754 mammal species (14\% of global mammal diversity) from 15 orders and 586 unique viral species (recognized viruses found in mammals) from 28 viral families. They used generalized additive models to identify and rank hostspecific predictors of the number of total and zoonotic viruses in mammals. The model showed that bats are host to a significantly higher proportion of zoonoses than other mammalian orders (after controlling for reporting effort and the other predictor variables). Moreover, the phylogenetic host breadth and other viral traits are significant predictors of zoonotic potential, providing a novel framework to assess if a newly discovered mammalian virus could infect people (Olival et al. 2017).

\section{Prediction of Origin and Intermediate Hosts}

Identifying the origin of the virus can help health authorities carry out accurate health surveillance. Undoubtedly, the development of genome sequencing technology has contributed a great deal to SARS-CoV-2 traceability. Wassenaar and Zou (2020) demonstrated that the noncoding flanks of the viral genome can be used to correctly separate the recognized four Betacoronavirus subspecies by whole-genome sequence comparisons, which has implications for rapid classification of new viruses. Comparing the sequence of the genomic sequence obtained with various mammalian coronavirus sequences suggests that bats may be a natural reservoir for SARS-CoV-2 (Paraskevis et al. 2020; Zhou et al. 2020), and Pangolins are potential intermediate hosts (Wong et al. 2020; Zhang et al. 2020).

Chen et al. (2020) used a low-input metagenomic nextgeneration sequencing (mNGS) approach on RNA extracted from bronchoalveolar lavage fluid (BALF) from two patients. The mNGS methodology used to investigate infectious microorganisms from original clinical samples is currently achievable (Gu et al. 2019). Firstly, they found that a majority of the viral reads $(99.9 \%$ and $99.7 \%$ respectively for sample 1 and 2) were associated with coronaviruses and identified SARS-CoV-2 as the sole pathogen. The genome comparisons indicated that SARSCoV-2 shared a $98.7 \%$ nucleotide identity with bat coronavirus strain $\mathrm{BtCoV} / 4991$ while it was quite divergent from SARS-CoV (79.7\%). Furthermore, phylogenetic trees

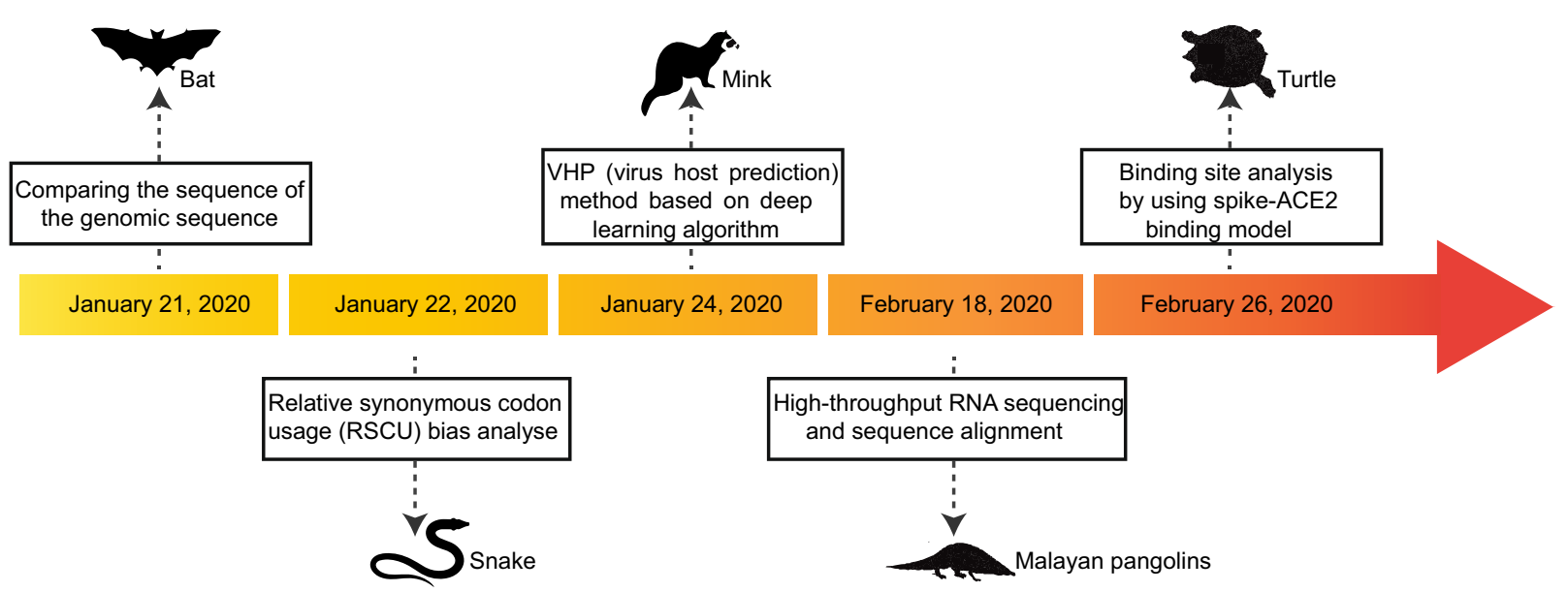

Fig. 1 Prediction of susceptible hosts, and date refers to the publication date of the preprint article. 
were reconstructed based on the nucleotide alignment of key viral genes by using the maximum likelihood method, employing a best fit substitution model and a SPR branch swapping algorithm. An understandable phylogeny showed SARS-CoV-2 has the most recent common ancestor with the neighboring bat coronaviruses, supporting the bat origin.

Another way to help identify the origin and evolutionary trends of viral hosts is to study codon usage bias given that the use of viral codons is subject to different selection pressures in different host environments. Ji et al. (2020) found that the codon usage bias of SARS-CoV-2 is most similar to that of snakes by using relative synonymous codon usage (RSCU) analyses. In fact, there are two levels of codon usage biases, one is at the amino acid level and the other is at the synonymous codon level. Amino acid composition can also introduce confounding effects if we only focus on the variation of synonymous codon usage. In order to overcome the above problem, another research team performed global correspondence analysis (CA), within-group correspondence analysis (WCA) and between-group correspondence analysis (BCA) among different genes in the coronavirus viral sequences ( $\mathrm{Gu}$ et al. 2020). The results showed that the SARS-CoV-2 had amino acid usage similar to bats, but the synonymous codon usages were relatively different, which indicated similar protein characteristics but maybe different evolutionary histories. However, these analytic approaches have not been used in studying viral sequences widely, so the information only provided some insights into wildlife reservoirs and that the further validation by animal model experiments is indispensable. Moreover, as a potential intermediated mammal, virus in civets share $\sim 99.8 \%$ genome identity to SARS-CoV in humans. As the origin for SARS-CoV-2 is most possibly the bats, and we should also be concerned about other mammals, while are likely to be predicted as intermediate hosts.

\section{Prediction Models in Transmission Dynamics Analysis}

In the case of a gradually improved infectious disease surveillance system, the research on forecasting and early warning of epidemics based on models has become the focus of the public health system. The principle aim is to establish a suitable mathematical model based on the dynamic characteristics of infectious disease transmission, and to conduct qualitative as well as quantitative analysis and computer simulations of the transmission process. Currently, the models used for forecasting and warning at home and abroad mainly include the time series model, the linear regression model, the grey dynamics model, the artificial neural network model, the Markov model, the Bayesian model and the Complex network model (Jennings et al. 2008; Zhu et al. 2011; Wesolowski and Suchacz 2012; Aghdam et al. 2013; Shen et al. 2013). Practice has proved that in public health emergencies, it is important to establish suitable prediction models to change passive prevention into active prevention. For example, during the outbreak of foot and mouth disease in the UK, the politicians relied heavily on mathematical modeling in their selection of epidemic control measures with great success (Ferguson et al. 2001; Keeling et al. 2001). In response to the current epidemic of SARS-CoV-2, many researchers have developed mathematical models with varying degrees of complexity, aiming to assess the capacity of pathogen transmission and which interventions are most likely to be effective (Fig. 2).

\section{Prediction of the Transmissibility of Pathogens}

In the early stages of the outbreak, it is important to gain an understanding of the transmission pattern and potential of SARS-CoV-2. If more than one secondary case is produced for each primary case on average, the chain of transmission events within an outbreak is extended. The basic reproduction number $\left(\mathrm{R}_{0}\right)$ is the most important index to measure and directly explain the level of virus transmission (Anderson et al. 2004). It refers to the average number of people who will be infected with an infectious disease under natural conditions. Due to the facts that (1) the infected patients might die, (2) some patients may develop immunity after recovering, and (3) that the infected population is of a limited size, the $\mathrm{R}_{0}$ value will decrease with the decrease in the infected population, and the transmission speed is significantly reduced (Anderson et al. 1992). Essentially, $\mathrm{R}_{0}$ determines how intensive a policy will need to be to control the epidemic, whereas both the generation time ( $\mathrm{Tg}$ ) and $\mathrm{R}_{0}$ determine the time available to implement suitably intensive controls.

One way to estimate $\mathrm{R}_{0}$ is to model an infectious disease curve that obeys exponential growth (De Silva et al. 2009). The nonlinear least square (NLS) framework has been adopted for data fitting and parameter estimation (Wallinga and Lipsitch 2007; Zhao et al. 2019). Since the transmission chain of SARS-CoV-2 remains unclear, many researches adopted the $\mathrm{Tg}$ information from SARS and MERS, which is similar to SARS-CoV-2. Zhao et al. (2020) reviewed the SARS-CoV-2 cases and suggests they might have been under-reported roughly from the 1 st to the 15 th of January 2020 because the number of cases appeared inconsistent with the following rapid growth of the epidemic curve since the 16th of January 2020. They modeled the epidemic curve of SARS-CoV-2 cases, in mainland China from December 1, 2019 to January 24, 


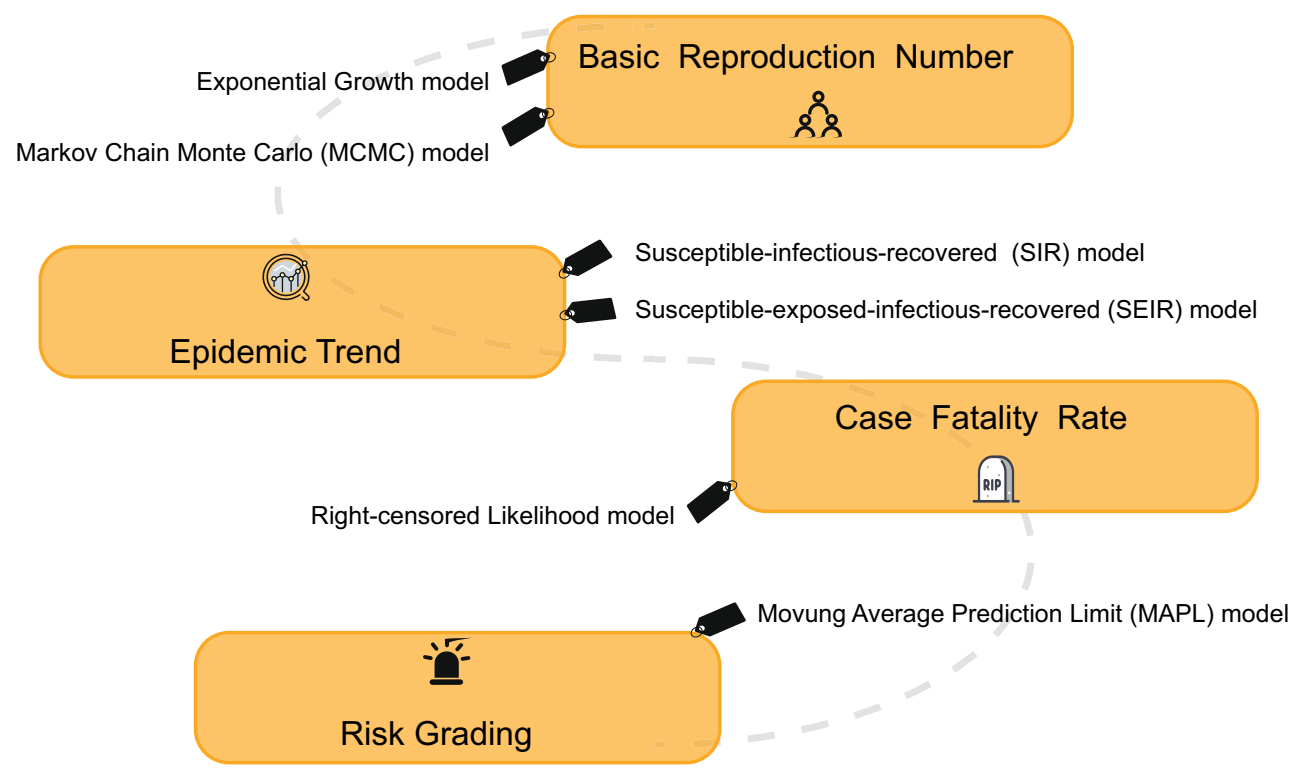

Fig. 2 Mathematical model for transmission dynamics analysis for macroscopic analysis.

2020 through the exponential growing Poisson process. The number of unreported cases was determined by the maximum likelihood estimation. As a result, they estimated the $\mathrm{R}_{0}$ of SARS-CoV-2 was 2.56 (95\% CI: 2.49-2.63) and the number of unreported cases was 469 (95\% CI: 403-540). This study helped us understand what might be happening in the early stages of outbreak. Another algorithm estimated $\mathrm{R}_{0}$ using the Markov Chain Monte Carlo (MCMC) method with Gibbs sampling and a non-informative flat prior. $\mathrm{Wu}$ et al. estimated the $\mathrm{R}_{0}$ for SARSCoV-2 was 2.68 (95\% CI: 2.47-2.86) (Wu JT et al. 2020a).

Although the $\mathrm{R}_{0}$ values estimated by different methods were different to some extent, it was not difficult to find that the $\mathrm{R}_{0}$ value remained larger than 1 at the early stage of the outbreak indicating the possibility of sustained human-to-human transmission ( $\mathrm{Li}$ et al. 2020). Adam et al. estimated that the median daily $\mathrm{R}_{0}$, declined from 2.35 (95\% CI: 1.15-4.77) 1 week before travel restrictions were introduced on January 23 to 1.05 (95\% CI: 0.413-2.39), which may have reflected the outbreak control efforts or a growing awareness of SARS-CoV-2 during this period (Kucharski et al. 2020).

\section{Prediction of the Super Spreading Event}

Population estimates of $\mathrm{R}_{0}$ can obscure considerable individual variation in infectiousness, as highlighted during the global emergence of SARS by numerous super spreading events in which certain individuals infected unusually large numbers of secondary cases (Dye and Gay 2003; Leo et al. 2003; Lipsitch et al. 2003; Riley et al. 2003; Shen et al. 2004; Bauch et al. 2005). While super spreading always remains a rare event, it can result in a large and explosive transmission event and have a major impact on the course of an epidemic.

For each primary case, Riou et al. generated secondary cases according to a negative-binomial offspring distribution with mean $\mathrm{R}_{0}$ and dispersion $\mathrm{k}$ (the lower the value of $\mathrm{k}$, the higher the impact of super spreading). After 1000 stochastic simulations for each individual combination, their simulations suggest that very low values of $\mathrm{k}$ are less likely, and the establishment of sustained transmission chains from single cases cannot be ruled out (Riou and Althaus 2020). Therefore, the author emphasized the importance of screening, surveillance and control efforts, such as early detection and isolation, contact tracing and the use of personal protective equipment.

\section{Prediction of the Trend of Epidemic}

The most classic infectious disease prediction and early warning models are the susceptible-infectious-recovered (SIR), susceptible-infectious-susceptible (SIS) and susceptible-infectious-recoverd-susceptible (SIRS) models. The SIR model is used to predict diseases in which individuals can obtain permanent immunity after infection. The SIS model is used for diseases in which the individual is not immune after the infection is cured. The SIRS model is used for diseases in which individuals can acquire immunity for a certain period of time after infection. However, these common predictive models are only applicable when there is a non-drug prevention intervention. The estimated numbers of infected people far exceed reported cases in the available literature which used these models 
(Anastassopoulou et al. 2020; Peng et al. 2020). So it is necessary to improve these traditional models according to the actual situations.

Joseph et al. used the susceptible-exposed-infectiousrecovered (SEIR) model to simulate the Wuhan city epidemic since it was detected in December, 2019 (Wu JT et al. 2020a). They estimated that 75,815 individuals (95\% CI: 37 304-130,330) individuals had been infected in Wuhan city as of January 25, 2020. And then the SEIR model was extended into a SEIR-metapopulation model to simulate the spread of SARS-CoV-2 across mainland China. Given that public health professionals and the general public have realized the threats caused by virus, the transmissibility of the epidemic might be reduced compared with its nascent stage at Wuhan city. So they assumed that the transmissibility of SARS-CoV-2 was reduced by $0 \%, 25 \%$, and $50 \%$ after Wuhan city was quarantined on January 23, 2020 and the rate of transmissibility was similar across all cities. The estimated result was that if there was no reduction in transmissibility, the Wuhan city epidemic would peak around April of 2020, and local epidemics across cities in mainland China would lag by $1-2$ weeks. A $50 \%$ reduction in transmissibility would push the $\mathrm{R}_{0}$ to 1.3 , in which case the epidemic would grow slowly without peaking during the first half of 2020. These analytical data gave us an early warning that SARS-CoV-2 could be about to become an epidemic in the absence of mitigation on its present trajectory.

The SIR structure was modified based on the unique characteristics of the outbreak of SARS-CoV-2. In the modified SIR model, recovered population $\mathrm{R}$ was extended to include those cured, died, and isolated in hospitals because under those conditions the virus does not spread (Zhu et al. 2020). This model was used to predict the trend under three possible scenarios, such as the current trend maintained, control efforts expanded, and person-to person contact increased due to work resuming. By March 12, 2020, the total infectious estimates in each of the three scenarios are $72,172,54,348$, and 149,774 . The model also predicted that the probability of no new cases in other cities at the end of February is $95.4 \%, 100 \%$, and $75.7 \%$, under these three scenarios respectively. The simulation suggested that strict quarantine of inner or inter-city population movement would have a significant effect on the suppression of virus spreading.

\section{Prediction of the Risk Grading}

The severe measures such as extending holidays and limiting group activities played an important role in stopping the spread of the epidemic. Significant results have been accompanied by high social costs. Therefore, in order to plan for the gradual recovery of social production activities after the epidemic is under control, it is essential to scientifically predict the future development of the epidemic.

In epidemiological studies, the moving average method is mostly used to provide early warning of infectious diseases (Petukhova et al. 2018). The main idea is to calculate based on the data of infectious disease incidence over a long period of time and then establish an early warning line. By comparing the incidence data of a certain period with the early warning line, the epidemic trend of the disease can be judged and the risk of incidence can be estimated. However, this method can only be applied to the outbreak warning of existing infectious diseases. To substitute for it, a moving average prediction limit (MAPL) method was proposed, its standard deviation can be used to establish a prediction limit to predict the epidemic trend, so as to timely predict the epidemic risk grading in the affected area. The data has shown that the number of new cases in the whole country has been moving towards a faster decline zone in the 11 days after February 12, 2020 outside Hubei province, and stabilized near the prediction limit of the faster decline zone in the 5 days up to February 23 , which is a low risk level. It indicates that after the nationwide anti-epidemic campaign, the epidemic situation in the country has been reduced as of the end of February. The overall trend of new suspected cases is consistent with the number of new confirmed cases. It is speculated that as long as the previous effective measures for outbreak prevention and control are adhered to, there is a great hope to keep the outbreak at a low risk level, and the risk of the epidemic in Hubei province in the future will be less serious. There is no doubt that understanding the real-time risk levels of the epidemic has a great guiding role in planning measures to gradually lift restrictions and restore normal economic production and social life.

\section{Prediction of the Case Fatality Rates of Diseases}

The term case fatality rate (CFR) is widely used to describe the proportion of infected people who eventually die from a disease caused by pathogens. Assessing CFR can help understand the severity of infection and anticipate the likely number of deaths by the end of the epidemic.

SARS-CoV-2 is the virus that can cause death in infected people. Jung et al. (2020) estimated the risk of death from confirmed cases (cCFR), while using data from confirmed cases outside mainland China and a right-censored likelihood to model the number of deceased cases and process the determination bias. The estimated cCFR value was $5.3 \%$ when the index case onset date was fixed on December 8, 2019 and 8.4\% when epidemic exponential growth occurred to fit the data with other model parameters. Although the cCFR of COVID-19 is not high compared to the 17\% CFR of SARS in Hong Kong (Donnelly 
et al. 2003; Ghani et al. 2005) and 20\% CFR of MERS in South Korea (Mizumoto et al. 2015), the 5\%-8\% risk of death is by no means negligible given the overall scale of the ongoing epidemic. However, the cCFR estimation models also have limitations, such as the cCFR only addressed fatality among confirmed cases. More precise infection fatality risk (IFR) estimates including infected individuals other than confirmed cases. In addition to quantifying the overall risk of death, future studies should identify groups at risk of death such as the elderly and potential comorbidities.

\section{Conclusions}

At present, in the context of global economic integration, countries around the world are getting close. On the one hand, human beings enjoy the convenience brought by economic development, and on the other hand, they bear the survival challenges brought by ecological destruction. With the continuous destruction of the environment and growth of population, human beings have been frequently attacked by infectious diseases. The COVID-19 epidemic caused much suffering, significant mortality, great disruption to social and work activities and considerable economic losses. To mitigate the spread of the virus, the Chinese Government has progressively implemented a metropolitan-wide quarantine of Wuhan city and several nearby cities since January 23, 2020. Numerous domestic airports and train stations, as well as international airports, have adopted temperature screening measures to detect individuals with fevers. Strict control measures are being implemented in densely populated and remote areas, including extended holidays, cancellation of crowd gatherings, calls for home isolation, and so on (Xiong and Yan 2020). Fortunately, with the efforts of the Chinese government and people, the outbreak has been brought under control relatively quickly.

The outbreak has not only caused huge losses, but also reminded us of the need to strengthen public health surveillance and management. At the micro level, it is important to find the agent that is the cause of observed morbidity and mortality (Gardy and Loman 2018). When used with phylogenetic analysis, it is possible to find the natural reservoir and intermediate host of the virus, which can help us isolate the source of the infection accurately. Furthermore, codon usage bias analysis provides us with another method and perspective to trace the source of the virus, although the results are still open to question. At the macro level, to solve the surveillance and detection problem, the next set of issues concern data capture, the development of diagnostic tests and treatment algorithms and the identification of public health measures to control the epidemic spread. Real-time data capture and associated analysis to reveal how the epidemic is expanding and how interventions are acting to slow its spread is essential. Based on the mathematical frameworks, we can better assess the infectious capacity of pathogens and the effects of public health control measures.

But the existing prediction models still have great limitations. First of all, the establishment of the model cannot be separated from the support of a large amount of data. In other words, the key to establish a reliable model is to track the epidemic dynamics and release the clinical information and epidemiological data in a timely manner. However, official data is often uncertain because medical resources are limited. The available data only reports confirmed cases in hospitals and ignores infected people who do not have access to medical services. This makes it difficult to accurately predict the development of the epidemic. Secondly, we need to make it clear that fitting all factors into a model is not easy and models are often built on strong assumptions so the data estimated by the model may be biased in some cases. In this case, simple public health measures are unlikely to be effective, and other measures are needed, such as tighter restrictions on movement, greater availability of antivirals drugs and expanded vaccine development and production facilities. These questions suggest that there is still much room for improvement in rapid risk assessment and accurate early warning of emerging infectious diseases. It is clearly in everyone's interest to greatly enhance global surveillance capabilities, especially in developing regions, and concomitantly to improve basic training in infectious disease and molecular epidemiology.

Acknowledgements JL is supported by grants from the National Science and Technology Major Project (2018ZX10101004) and the Youth Innovation Promotion Association of CAS (2019091).

\section{Compliance with Ethical Standards}

Conflict of interest The authors declare that they have no conflict of interest.

Animal and Human Rights Statement This article does not contain any studies with human or animal subjects performed by any of the authors.

\section{References}

Aghdam R, Pezeshk H, Malekpour SA, Shemehsavar S, Eslahchi C (2013) A clustering approach for estimating parameters of a profile hidden Markov model. Int J Data Min Bioinform 8:66-82 Anastassopoulou C, Russo L, Tsakris A, Siettos C (2020) Data-based analysis, modelling and forecasting of the novel coronavirus (2019-nCoV) outbreak. medRxiv 
Anderson RM, Anderson B, May RM (1992) Infectious diseases of humans: dynamics and control. Oxford University Press, Oxford

Anderson RM, Fraser C, Ghani AC, Donnelly CA, Riley S, Ferguson NM, Leung GM, Lam TH, Hedley AJ (2004) Epidemiology, transmission dynamics and control of SARS: the 2002-2003 epidemic. Philos Trans R Soc Lond Ser B Biol Sci 359:1091-1105

Bauch CT, Lloyd-Smith JO, Coffee MP, Galvani AP (2005) Dynamically modeling SARS and other newly emerging respiratory illnesses: past, present, and future. Epidemiology 16:791-801

Chen L, Liu W, Zhang Q, Xu K, Ye G, Wu W, Sun Z, Liu F, Wu K, Zhong B (2020) RNA based mNGS approach identifies a novel human coronavirus from two individual pneumonia cases in 2019 Wuhan outbreak. Emerg Microb Infect 9:313-319

Cheng ZJ, Shan J (2020) 2019 Novel coronavirus: where we are and what we know. Infection 48:1-9

De Silva U, Warachit J, Waicharoen S, Chittaganpitch M (2009) A preliminary analysis of the epidemiology of influenza A (H1N1) $\mathrm{v}$ virus infection in Thailand from early outbreak data, June-July 2009. Eurosurveillance 14:19292

Donnelly CA, Ghani AC, Leung GM, Hedley AJ, Fraser C, Riley S, Abu-Raddad LJ, Ho L-M, Thach T-Q, Chau P (2003) Epidemiological determinants of spread of causal agent of severe acute respiratory syndrome in Hong Kong. Lancet 361:1761-1766

Dye C, Gay N (2003) Modeling the SARS epidemic. Science 300:1884-1885

Ferguson NM, Donnelly CA, Anderson RM (2001) Transmission intensity and impact of control policies on the foot and mouth epidemic in Great Britain. Nature 413:542-548

Gardy JL, Loman NJ (2018) Towards a genomics-informed, realtime, global pathogen surveillance system. Nat Rev Genet 19:9

Ghani A, Donnelly C, Cox D, Griffin J, Fraser C, Lam T, Ho L, Chan W, Anderson R, Hedley A (2005) Methods for estimating the case fatality ratio for a novel, emerging infectious disease. Am J Epidemiol 162:479-486

Gu W, Miller S, Chiu CY (2019) Clinical metagenomic nextgeneration sequencing for pathogen detection. Annu Rev Pathol Mech Dis 14:319-338

Gu H, Chu DK, Peiris JSM, Poon LL (2020) Multivariate analyses of codon usage of SARS-CoV-2 and other betacoronaviruses. bioRxiv

Huang C, Wang Y, Li X, Ren L, Zhao J, Hu Y, Zhang L, Fan G, Xu J, Gu X (2020) Clinical features of patients infected with 2019 novel coronavirus in Wuhan, China. Lancet 395:497-506

Jennings LC, Monto AS, Chan PK, Szucs TD, Nicholson KG (2008) Stockpiling prepandemic influenza vaccines: a new cornerstone of pandemic preparedness plans. Lancet Infect Dis 8:650-658

Ji W, Wang W, Zhao X, Zai J, Li X (2020) Cross-species transmission of the newly identified coronavirus 2019-nCoV. J Med Virol 92:433

Jung S-M, Akhmetzhanov AR, Hayashi K, Linton NM, Yang Y, Yuan B, Kobayashi T, Kinoshita R, Nishiura H (2020) Real-time estimation of the risk of death from novel coronavirus (COVID19) infection: inference using exported cases. J Clin Med 9:523

Karesh WB, Dobson A, Lloyd-Smith JO, Lubroth J, Dixon MA, Bennett M, Aldrich S, Harrington T, Formenty P, Loh EH (2012) Ecology of zoonoses: natural and unnatural histories. Lancet 380:1936-1945

Keeling MJ, Woolhouse ME, Shaw DJ, Matthews L, Chase-Topping M, Haydon DT, Cornell SJ, Kappey J, Wilesmith J, Grenfell BT (2001) Dynamics of the 2001 UK foot and mouth epidemic: stochastic dispersal in a heterogeneous landscape. Science 294:813-817

Kucharski AJ, Russell TW, Diamond C, Liu Y, Edmunds J, Funk S, Eggo RM, Group Cnw (2020) Early dynamics of transmission and control of COVID-19: a mathematical modelling study. medRxiv

Leo Y, Chen M, Heng B, Lee C (2003) Severe acute respiratory syndrome-Singapore, 2003. MMWR Morb Mortal Wkly Rep $52: 405$

Li Q, Guan X, Wu P, Wang X, Zhou L, Tong Y, Ren R, Leung KS, Lau EH, Wong JY (2020) Early transmission dynamics in Wuhan, China, of novel coronavirus-infected pneumonia. N Engl J Med 382:1199-1207

Lipsitch M, Cohen T, Cooper B, Robins JM, Ma S, James L, Gopalakrishna G, Chew SK, Tan CC, Samore MH (2003) Transmission dynamics and control of severe acute respiratory syndrome. Science 300:1966-1970

Mizumoto K, Saitoh M, Chowell G, Miyamatsu Y, Nishiura H (2015) Estimating the risk of Middle East respiratory syndrome (MERS) death during the course of the outbreak in the Republic of Korea, 2015. Int J Infect Dis 39:7-9

Morgan O (2019) How decision makers can use quantitative approaches to guide outbreak responses. Philos Trans R Soc B 374:20180365

Olival KJ, Hosseini PR, Zambrana-Torrelio C, Ross N, Bogich TL, Daszak P (2017) Host and viral traits predict zoonotic spillover from mammals. Nature 546:646-650

Paraskevis D, Kostaki EG, Magiorkinis G, Panayiotakopoulos G, Sourvinos G, Tsiodras S (2020) Full-genome evolutionary analysis of the novel corona virus $(2019-\mathrm{nCoV})$ rejects the hypothesis of emergence as a result of a recent recombination event. Infect Genet Evol 79:104212

Peng L, Yang W, Zhang D, Zhuge C, Hong L (2020) Epidemic analysis of COVID-19 in China by dynamical modeling. arXiv preprint arXiv:200206563

Petukhova T, Ojkic D, McEwen B, Deardon R, Poljak Z (2018) Assessment of autoregressive integrated moving average (ARIMA), generalized linear autoregressive moving average (GLARMA), and random forest (RF) time series regression models for predicting influenza A virus frequency in swine in Ontario. Canada. PloS One 13:e0198313

Riley S, Fraser C, Donnelly CA, Ghani AC, Abu-Raddad LJ, Hedley AJ, Leung GM, Ho L-M, Lam T-H, Thach TQ (2003) Transmission dynamics of the etiological agent of SARS in Hong Kong: impact of public health interventions. Science 300:1961-1966

Riou J, Althaus CL (2020) Pattern of early human-to-human transmission of Wuhan 2019 novel coronavirus (2019-nCoV), December 2019 to January 2020. Eurosurveillance 25:2000058

Shen Z, Ning F, Zhou W, He X, Lin C, Chin DP, Zhu Z, Schuchat A (2004) Superspreading sars events, Beijing, 2003. Emerg Infect Dis 10:256

Shen X, Ou L, Chen X, Zhang X, Tan X (2013) The application of the grey disaster model to forecast epidemic peaks of typhoid and paratyphoid fever in China. PLoS ONE 8:e60601

Wallinga J, Lipsitch M (2007) How generation intervals shape the relationship between growth rates and reproductive numbers. Proc R Soc B Biol Sci 274:599-604

Wassenaar TM, Zou Y (2020) 2019_nCoV: rapid classification of betacoronaviruses and identification of traditional Chinese medicine as potential origin of zoonotic coronaviruses. Lett Appl Microbiol. https://doi.org/10.1111/lam.13285

Wesolowski M, Suchacz B (2012) Artificial neural networks: theoretical background and pharmaceutical applications: a review. J AOAC Int 95:652-668

Wong MC, Cregeen SJJ, Ajami NJ, Petrosino JF (2020) Evidence of recombination in coronaviruses implicating pangolin origins of nCoV-2019. bioRxiv

Wu JT, Leung K, Leung GM (2020a) Nowcasting and forecasting the potential domestic and international spread of the 2019-nCoV 
outbreak originating in Wuhan, China: a modelling study. The Lancet 395:689-697

Wu P, Hao X, Lau EH, Wong JY, Leung KS, Wu JT, Cowling BJ, Leung GM (2020b) Real-time tentative assessment of the epidemiological characteristics of novel coronavirus infections in Wuhan, China, as at 22 January 2020. Eurosurveillance 25. https://doi.org/10.2807/1560-7917

Xiong H, Yan H (2020) Simulating the infected population and spread trend of 2019-nCov under different policy by EIR model. Available at SSRN 3537083

Xu X, Chen P, Wang J, Feng J, Zhou H, Li X, Zhong W, Hao P (2020) Evolution of the novel coronavirus from the ongoing Wuhan outbreak and modeling of its spike protein for risk of human transmission. Sci China Life Sci 63:1-4

Zhang Z, Wu Q, Zhang T (2020) Pangolin homology associated with 2019-nCoV. bioRxiv

Zhao S, Musa SS, Fu H, He D, Qin J (2019) Simple framework for real-time forecast in a data-limited situation: the Zika virus
(ZIKV) outbreaks in Brazil from 2015 to 2016 as an example. Parasit Vectors 12:344

Zhao S, Musa SS, Lin Q, Ran J, Yang G, Wang W, Lou Y, Yang L, Gao D, He D (2020) Estimating the unreported number of novel coronavirus (2019-nCoV) cases in China in the first half of January 2020: a data-driven Modelling analysis of the early outbreak. J Clin Med 9:388

Zhou P, Yang X-L, Wang X-G, Hu B, Zhang L, Zhang W, Si H-R, Zhu Y, Li B, Huang C-L (2020) Discovery of a novel coronavirus associated with the recent pneumonia outbreak in humans and its potential bat origin. BioRxiv

Zhu M, Zu R, Huo X, Bao C, Zhao Y, Peng Z, Yu R, Shen H, Chen F (2011) The application of time series analysis in predicting the influenza incidence and early warning. Zhonghua yu fang yi xue za zhi [Chin J Prev Med] 45:1108-1111

Zhu X, Zhang A, Xu S, Jia P, Tan X, Tian J, Wei T, Quan Z, Yu J (2020) Spatially explicit modeling of 2019-nCoV epidemic trend based on mobile phone data in Mainland China. medRxiv 